\title{
Hybrid presence
}

\section{Integrating interprofessional interactions with digital consultations}

\section{Line Maria Simonsen}

Department for the Study of Culture, University of Southern Denmark

\begin{abstract}
Healthcare practitioners struggle to adapt to the changes that new digital media entail for social interactions, but what does the struggle look like, and how is it embedded in these professionals' everyday experiences? I investigate these questions in this study of how digitalisation conditions social interactions in the context of the Danish medical setting by drawing on ethnographic work. Moreover, via a video-recorded case study, this article shows how two practitioners organise social actions by exploiting features of a digital communication system in a situation where they manage a practical problem. I propose the concept of hybrid presence related to the scientific fields of dialogism and distributed cognition as an explanation of how the participants are capable of immersing themselves with both the digital technology and the social interaction. Hybrid presence thus proves useful in the discussion of how practitioners may struggle with technology.
\end{abstract}

Keywords: hybrid presence, digital consultations, absent presence, dialogism, cognitive ethnography

\section{Introduction}

Healthcare technology shapes the way we understand our bodies, how we define disorders, and how healthcare practitioners make treatments in practices of healthcare (see Mol, 2002; Oudshoorn, 2009). Accordingly, technologies are not passive devices that just facilitate medical actions: they deeply saturate how we interact socially and make meaning with each other and in the world. One critical view of the comprehensive implications that technology may entail for meaning-making and coherence in social interactions has been conceptualised as "absent presence" by Gergen (2002). Absent presence is described as a technology-induced phenomenon that problematises how communication technologies divide one's physical presence between the technology being used and the social interaction. In short, when we use technology in the presence of each other, we are indeed physically present, but we are simultaneously absent as we are absorbed in the technologically mediated world (Gergen, 2002). ${ }^{1}$

Simonsen, L. M. (2021). Hybrid presence: Integrating interprofessional interactions with digital consultations. Nordicom Review, 42(S4), 22-44. https://doi.org/10.2478/nor-2021-0039 
Recently, Baym (2015) uses Gergen's (2002) notion of absent presence to contextualise how mediated interactions are ambiguous in nature. Although they may enable us to extend the borders of our social worlds, they can also cause struggles with the new forms of interpersonal connection, digital control, and constrained autonomy they simultaneously entail (Baym, 2015). The ambiguous character of digital media and mediated interactions thus makes us struggle in how we enact, sustain, or establish a coherent presence in social interactions while we use technology. Moreover, a study on mobile devices (Aagaard, 2016) found that absent presence can result in specific disintegrated microsocial dynamics: a lack of eye contact, mechanical intonations, and delayed responses and motionless body language. These implications of absent presence on social interactions thus manifest as a direct problem, because these fragmented microsocial dynamics in face-to-face encounters impair the degree of interpersonal certainty that people experience (Aagaard, 2016). Overall, technology may divide our ways of engaging with each other and thus makes us struggle in how we direct our attention and integrate presence in situ.

On the one hand, Gergen's (2002) conceptualisation of absent presence denotes a significant problem in terms of divided presence between people, and thus, inhibiting connection, trust, and depth in social interactions (Aagaard, 2016; Baym, 2015; Gergen, 2002). Yet, on the other hand, working with technology during social interactions is an everyday practice for various healthcare professionals (e.g., Greatbatch et al., 1993, 1995; Luff \& Heath, 2019; Nielsen, 2019). In the context of Danish primary healthcare, I conducted an ethnographic study focusing on the use, production, and interpretation of digital consultations (Simonsen, in progress). This study suggests that healthcare practitioners are skilled at working with digital technology while engaging with other agents because they often are able to incorporate multiple contexts and sociocultural resources (e.g., roles, institutional procedures, and structures) as part of organising integrated social interactions that are conducive for medical task-performance (e.g., patient treatment). The observation of these everyday experiences challenges the idea that healthcare practitioners should struggle with technology-induced absent presence. In this article, I therefore propose the concept "hybrid presence" to explain practitioners' abilities to achieve a coherent and collaborative social interaction. In short, the concept of hybrid presence denotes how practitioners might integrate rather than fragment interactions due to how they invoke multiple resources afforded by the technology in use (e.g., in verbalising technical features) and by aligning these with the needs emerging in the social interaction (e.g., through requests and problem-solving pertaining to a plan or a patient). Therefore, I ask the following questions: How does hybrid presence emerge? What does it look like in a case of an interprofessional interaction? How is it achieved? What are the consequences with regard to task performance?

My overall aim in this article is to empirically explore and scrutinise in depth how examples of everyday experiences are organised in social interactions involving technology during which health practitioners achieve hybrid presence, and subsequently how these challenge Gergen's (2002) problematisation, which is valuable in the discussion of what may cause practitioners to struggle with technology.

Following this introduction, I provide some background regarding recent sociotechnological advancements in Danish healthcare, then I briefly introduce the theoretical framework that presents the insights from which I draw the conceptualisation of hybrid 
presence. Next, the methods and analytical approach are introduced, followed by the empirical findings and then the validity and adequacy of both hybrid and absent presence for interactions are discussed. Finally, the conclusion summarises how research communities can benefit from the analytical findings, thus suggesting a direction for future studies.

\section{Background: Digitalising the Danish primary healthcare sector}

In the pursuit of making the Danish primary healthcare sector more time and cost-efficient, in 2009 the Danish government made it mandatory for all general practitioners to implement and use digital consultations. ${ }^{2}$ Since digital consultation technology enables patients and healthcare practitioners to exchange written messages, it transforms medical practice, because it reduces the number of physical consultations some patients would have had with their general practitioner. In technical terms, this means that digital consultations, rather than promoting face-to-face interactions, instead mediate interactions via semiotic and symbolic features (e.g., written language and visual images) that are exchanged digitally. Accordingly, digital consultation technology shapes new ways of engaging, as well as new meanings and action potentials, for healthcare practitioners on at least two task levels. At an activity level, for example, when the agent interpersonally (or rather interdigitally) engages in the situated process of writing and reading of correspondences, she or he engages with digital representations instead of real-world embodied interactions, which requires different sensitivities with regard to, for instance, writing style and interpretation. At an organisational level, more administrative work - such as the managerial work that coordinates and ensures the execution of the activity level - is imposed on healthcare practitioners. As some digital consultations may require immediate response, for instance, in the case of incoming (critical) test results, practitioners must take into account how they organise their engagement with the digital system, so the critical correspondences can be acted on right away, and not get lost in the inbox. The organisational task level, thus, includes activities of monitoring the digital consultations and requires coordination of who is monitoring them. Therefore, both levels - responding to digital consultations and the administration of digital consultations - constitute important tasks in the overall organisation of patient care, treatment, and interprofessional collaboration.

Current studies (Assing Hvidt et al., 2020; Fage-Butler \& Jensen, 2015; Grønning, 2016; Grønning et al., 2020; Laursen et al., under review) have described advantages and disadvantages of digital consultations pertaining to the medium's technological features, analysed the content of digital consultation correspondences, and discussed mediated relational aspects of patient-doctor dyads. Though, while these studies provide important insights of phenomenological value, little is known about how digital consultations condition social interactions when they are used, produced, and interpreted in practitioner-practitioner dyads as an embedded part of the practitioners' everyday working life. Subsequently, the situated manifestation of the activity and organisational task level is underexplored. However, as part of conducting the ethnographic study of digital consultations, I investigated how practitioners use digital consultations in their everyday interprofessional interactions (Simonsen, in progress). My study suggests that the practitioners' use of digital consultations in some cases challenges Gergen's (2002) conceptualisation of absent presence, wherefrom the concept of hybrid presence proves 
useful. Accordingly, this article, on the one hand, derives the concept of hybrid presence based on empirical observations from the ethnographic study, and on the other, it employs and adapts the concept of hybridity from its scientific recognition found in a dialogical framework combined with insights from a distributed cognitive perspective (below) in line with recent ideas related to hybrid cognition (Simonsen \& Steffensen, 2019, 2021/in press).

Before I introduce the theoretical insights related to hybridity, two remarks are subsequently important to make. First, as the article is concerned with the empirical recognition and understanding of hybrid presence, it is relevant to emphasise that though hybrid presence and hybrid cognition (Simonsen \& Steffensen, 2019) are interrelated and originate from the same theoretical insights, hybrid cognition is rather an explanatory framework, whereas hybrid presence add an empirical emphasis by designating the practitioners' ability to enact coherent presence while working with the digital technology at the same time as they engage in the social interaction (Simonsen \& Steffensen, 2021/ in press). Second, though the theoretical insights of dialogism and distributed cognition offer a strong position in line with media studies' interests for the investigation of the organisation and influence of technological artefacts (e.g., Latour, 2000; Scolari, 2012), it is beyond the scope of this article to provide an exhaustive overview of the scientific fields of dialogism and distributed cognition, and related branches, such as human interactivity (e.g., Steffensen, 2013). Therefore, the following theoretical introduction is only modest and brief, and for further overview, I refer to the work of Linell and colleagues (Linell, 2009, 2010, 2017; Linell \& Thunqvist, 2003), Hutchins (1995, 2014), Sutton (2012), Steffensen $(2013,2015)$, and Trasmundi (2020).

\section{Insights from a dialogical framework and a distributed cognitive perspective}

As noted by Simonsen \& Steffensen (2019), the idea of tracing hybridity to social interactions comes from the insights designated by Linell and collaborators (e.g., Linell \& Thunqvist, 2003). In Linell's dialogical framework "hybridity" is defined as "a property found in most communication activity types [...] that is a mixture of different forms and functions, as well as influences from other communicative genres and activity types" (Linell, 2009: 211). Accordingly, I use the concept of hybridity in a similar vein as I, like Linell's work within the study of interactions and language (Linell, 2009; Linell \& Thunqvist, 2003), scrutinise the emergence of hybridity in social interactions. Paying attention to hybridity allows the researcher to view the phenomenon under investigation with a new perspective; for instance, a police interrogation might also be understood as a form of social education or therapy (Linell \& Thunqvist, 2003). By building on Goffman's work related to how individuals organise experience (Goffman, 1974, 1983) and notions of interactional hybridity found in communication theory (Sarangi, 2000), Linell's employment of hybridity refers to how individuals can attune themselves to multiple, sometimes conflicting, goals and activities at the same time in the same situation (Linell \& Thunqvist, 2003; see Simonsen \& Steffensen, 2019). This may be evident in the way individuals invoke or relate to "third parties", that is, in orientations to, for instance, media, organisational procedures, artefacts, and other persons that are not directly present in the interpersonal interaction (Linell, 2017). 
A dialogical framework thus recognises interactional complexity as third parties can be invoked in dialogical orientations at all times. In the dialogical framework's pursuit of understanding such interactional complexity and multiplicity (as in "multivoicedness", traced to Bakhtin, 1981), the researcher is encouraged to explore the situated dialogue and interaction beyond the mere investigation of the talk-in-interaction occurring between copresent agents by regarding how, for instance, past histories and institutional normativities (i.e., third parties) condition the organisation of the social interaction. Thus, employing a dialogical framework offers a way to understand sociocultural and cognitive dimensions, as it recognises how verbal utterances and interactions "with material environments as well as more abstract contexts" (Linell, 2017: 303) are constitutive processes of human sense-making (Linell, 2009, 2017). In line with this thinking, human interactivity, defined as "sense-saturated coordination that contributes to human action [emphasis original]" (Steffensen, 2013: 196), importantly recognises how situated interactions are "saturated" by means of, for instance, participants' dialogical orientations to third parties. Interactivity research promotes interests for the multi-temporal scales by which human interaction are constrained. In this view, both dialogism and human interactivity deals with complexities that are observable in interactions yet also extends the analysis to domains beyond the situated activity. Such insights are closely related to a distributed perspective on cognition (e.g., Hutchins, 1995, 2014; Pedersen \& Steffensen, 2014; Sutton, 2012). A distributed perspective on cognition promotes investigations of how cognitive processes (like sense-making and problem-solving) and hence, actions, are organised through multiple interacting parts, and thus presents a systemic understanding of cognition. For instance, a cognitive process, like problem-solving, might be reorganised due to an agent's usage of artefacts, sociocultural resources (e.g., verbal patterns, organisational norms), and interactions with other agents. As such, a distributed perspective on cognition recognises the following:

Many cognitive states and processes are hybrids, unevenly distributed across the physical, social, and cultural environments as well as bodies and brains, hooking up in both temporary and more enduring ways with other people and with certain things - artifacts, media, technologies, or institutions - each with its own history and tendencies. (Tribble \& Sutton, 2011: 95)

Cognition, then, is not viewed just as an intercranial process, rather, it is understood as distributed across artefacts, members of different social groups, and time in the sense that "products of earlier events can transform the nature of later events" (Hollan et al., 2000: 176). In combining the dialogical understanding of hybridity from Linell (2009; Linell \& Thunqvist, 2003) with notions of cognitive processes and states as hybrids, hybrid presence, as conceptualised in this article, explains a form of distributed cognition. Though all dialogical and cognitive processes have the potential to be hybrid, not all dialogical and cognitive processes necessarily lead to hybrid presence. For example, if two nurses are managing digital consultations and at the same time conversing about what is for lunch, the mere presence of a technological artefact and a smooth ongoing conversation does not constitute hybrid presence by default. Instead, hybrid presence may be the case if the two participants integrate the social interaction with the use of the technology. For example, if one nurse uses information from the digital consultations in the conversation, and the second nurse becomes involved in the conversation 
with attention to affordances that might emerge from both the social interaction (e.g., concerned micro-expressions, like intonation and gestures) as well as the digital consultation (e.g., the content and writing style of the message), then both nurses engage in the social interaction in a way that can be explained via hybrid presence. This is the case because their presence is coherently balanced by how the technology in use is integrated with the verbal and embodied cues emerging from the social interaction that unfolds.

In sum, hybrid presence refers to a particular dialogical-cognitive ability that enables agents to act in a way that is conducive for their task-performance, because they integrate the social interaction with the situated use of technology.

It is worth noting that other studies have employed dialogical approaches in investigations of medical domains and similarly connected their studies with a distributed cognitive perspective (e.g., Cowley et al., 2010; Pedersen, 2015; Pedersen \& Steffensen, 2014; Simonsen \& Steffensen, 2019; Steffensen, 2012; Trasmundi, 2020). Bridging the two disciplines allows one to study the complex and multi-scalar dynamics of human interactivity (see Gahrn-Andersen et al., 2019; Steffensen, 2013), which contributes to detailed analyses of, for instance, how material artefacts aid or prohibit dialogical behaviour (Pedersen \& Steffensen, 2014). These studies entail real-world observations enabled by, for example, cognitive ethnographic methods (Alač \& Hutchins, 2004; Becvar et al., 2005; Hutchins, 1995; Trasmundi, 2020). Similarly, for this article I have collected data via cognitive ethnography and furthermore analysed the data via cognitive event analysis (CEA). In the following section, I briefly unfold the methods and present the analytical findings.

\section{Data and methods}

\section{Cognitive ethnography and data}

This article draws on data from an ethnographic study. In collecting the data, methods from cognitive ethnography (Becvar et al., 2005; Hollan et al., 2000; Hutchins, 1995, 2014) were employed. Cognitive ethnography differs from traditional ethnography in its interest in cognitive processes, like problem-solving and decision-making. Moreover, as it has been developed along the insights related to the distributed cognitive perspective, it holds a methodological focus on cognitive events: "We are interested not only in what people know, but in how they go about using what they know to do what they do" (Hollan et al., 2000: 179). This means that the cognitive ethnographer interdependently combines the traditional fieldwork of participant observations with microlevel analysis of cognitive events and practices (Alač \& Hutchins, 2004). Accordingly, I have conducted an ethnographic study comprising participant observations with and without video recordings of healthcare practitioners' everyday working lives. In order to elicit in-depth knowledge about the organisation of interprofessional interactions with digital consultation technology, I conducted the study in collaboration with both a general practice and a nursing home over a ten-month period (2018-2019). ${ }^{3}$ Nine days of fieldwork were conducted in the general practice, which was administered by three general practitioners, and five days of fieldwork were conducted at the nursing home. Face-to-face interactions between practitioners (doctors, nurses, and care assistants) and between practitioners and patients, as well as the practitioners' work with digital consul- 
tations, were observed. Three formal semistructured interviews with the doctors were conducted as were additional informal interviews with all practitioners, and fieldnotes were collected throughout the fieldwork. In total, the data consists of over 20 hours of video recordings, 60 pages of fieldnotes, and approximately 8 hours of audio recordings. This ethnographic material provided an overall and necessary background for identifying and analysing what hybrid presence looks like in interprofessional interactions. In the following, I focus on one case study that informs us of hybrid presence, and it will be analysed through the lens of CEA (Gahrn-Andersen et al., 2019; Steffensen, 2013; Steffensen et al., 2016).

\section{Cognitive event analysis}

Overall, CEA is an event-focused and qualitative method for analysing video-recorded data at a microlevel, where changes in affordances (Chemero, 2000; Gibson, 1986) are scrutinised. CEA is thus a method for the study of human interactivity, as it allows the researcher to study the fine-grained interactional dynamics that emerge and constrain an agent and their environment, as a cognitive event transpires (Steffensen et al., 2016). Accordingly, the researcher works backwards from identifying an interactional change to scrutinising what prompted the event to emerge as it did by locating the pivotal preconditions (in CEA terms, "event pivots") for a decision being made or a problem being solved. To do this, CEA combines insights from the overall ethnographic fieldwork with microlevel interactional and linguistic analyses in line with multimodal interaction analysis (Goodwin, 2013; Linell, 2009; Mondada, 2018; Norris, 2004).

Procedurally, CEA spans five steps (see Gahrn-Andersen et al., 2019; Steffensen, et al., 2016 for elaboration and examples): 1) a cognitive event identification, for example, identifying a change in the interaction, such as a problem being solved; 2) an event pivot identification, for example, identifying salient transitions that prompted the change in the interaction, such as when the agents realise there is a problem, when they search for a solution, and if and when they solve the problem; 3) data annotation, for example, segmenting and annotating the video-recorded data in order to scrutinise the verbal and intercorporeal coordination that made the problem emerge the way it did; 4) cognitive trajectory segmentation, for example, the video sequence is segmented into "functionally and/or behaviourally defined phases [emphasis original]" (Steffensen et al., 2016: 84); and 5) the cognitive trajectory analysis, where the researcher investigates how the event pivot, for example, solving the problem, is enabled based on interactional phase transitions and coordination of the agents' verbal and other relevant bodily orientations.

The CEA outline and structure in this article is deeply inspired by the insights and work of Trasmundi and Steffensen (e.g., 2016) and follow a similar approach with regard to data selection, figure presentations, and procedural rigor. In the following section, exemplary findings from the ethnographic study are described first on an empirical level and then on a theoretical level with regard to the conceptualisation of hybrid presence. After the introduction of the findings that exemplify aspects of the practitioners' everyday experiences and actions related to the use of digital consultations, I zoom in on the video-recorded case study to scrutinise what hybrid presence looks like in verbal and bodily coordination in an interaction between two practitioners. 


\section{Everyday use of digital consultations}

\section{A cognitive ethnography: Finding hybrid presence}

One key finding of how practitioners use, produce, and interpret digital consultations concerns that practitioners habitually collaborate around computers in breakrooms, in the clinic at the general practice, in the ward at the nursing home, and in different office spaces in both locations. For example, the nurses at the nursing home plan their day in the morning while sitting in front of their computers in the office. During these planning activities, they make notes on printouts, adjust treatment plans, and read and reply to digital consultations on their computers while simultaneously conversing with each other. These conversations comprise small talk, discussions of patients and treatments, and the administration of tasks to be conducted during the day in ways that intermesh the activities of reading and writing digital consultations with organisational tasks related to who and when the messages need, say, a response. In these situations, two nurses would often sit at computers placed back-to-back, and while one nurse could read and write digital consultations and coordinate plans based on the overview and changes they may provide, the other nurse might manage medicine dosages if not also engaged with the digital consultations. Either way, the nurses had become highly skilled in interacting with the digital system while responding to one another, as they would often exploit information from the digital consultations to solve problems and coordinate tasks. Thus, the ongoing collaboration between the nurses adapted, as they were able to engage in the social interaction while paying attention to the digital consultations on the computer screen. As such, I noticed how much of the social organisation between the practitioners was integrated with their technology use. Hence, absent presence (Gergen, 2002) did not seem to appear as a struggle that impeded their social interaction.

The same collaborative pattern was also observed at the general practice, where three doctors, two secretaries, and two nurses would stand around the computer and help each other with, for instance, finding the right technical feature or payment code on the screen. They could stand next to each other with equal access to the computer screen in the clinic's different offices and in the breakroom. They would also interact with unequal visual access to the screen, such as when one of the doctors would manage digital consultations on a laptop in the breakroom. Similar to the situation at the nursing home, the doctors in the general practice would often incorporate information from digital consultations as part of the social interaction. Even when they had unequal visual access, the doctor engaged with the digital consultations could engage in interactions with copresent others and the digital system. For example, the doctors would verbally and gesturally invoke medical or organisational information from a digital consultation in the interaction with each other. Here, they could use the correspondences to question and qualify their medical judgement of a patient's treatment if they had doubts about the patient's request, and they could, for instance, also adapt organisational planning in the clinic with regard to who should go on a medical home visit. Hence, the doctors, instead of fragmenting their interpersonal engagement due to media absorption, as suggested by Gergen (2002), rather relied on the hybridity emerging between the digital consultation and the social interaction in the situation. Because they were able to dialogically invoke information afforded by the digital system (e.g., test results) and integrate it with the behavioural needs emerging from the social interactions (e.g., asking ques- 
tions and solving problems), they would expand their task-performance by exploiting the resources from both the social and technological domains. For example, one doctor using a laptop in the breakroom might question a colleague about a patient from whom he had received a digital consultation. Based on the dialogical invocation of the digital consultation integrated with the doctor's attention to his colleague, he would smoothly engage in this hybrid interaction with his colleague. The colleague, who did not have visual access to the laptop, would engage in the interaction in a way that supported a joint collaboration. Similarly, other issues, such as questions and medical judgements, could be handled in collaboration related to the hybridity between the digital technology and the copresence of the doctors, as the social interaction unfolded.

\section{Circumventing the struggle of absent presence with hybrid presence}

Based on these ethnographic findings of observed interactional dynamics, it appears that health practitioners ${ }^{4}$ in some cases collaboratively adapt their social interactions while using digital consultation technology. Because the practitioners engage in the social interaction in a way that integrates both features of the digital consultation system and the embodied medical work that aids them in coordinating interpersonal, medical, and administrative issues, I propose the term hybrid presence for explaining these everyday experiences. The concept is useful for explaining what happens when the practitioners, in contradiction to Gergen's (2002) critique, indeed exploit the hybridity of the presence conducive for the interaction, instead of impairing it. Hybrid presence denotes instances where practitioners skilfully integrate technological engagement as part of their social interaction without impeding it. The concept of hybrid presence comes from identifying these cases of integrated interactions with technology across the ethnographic material, and it aids the knowledge elicitation of overall organisational and interpersonal usages of the digital consultations. However, due to the complexity of hybrid presence, it is necessary to identify and examine what it looks like in an interprofessional interaction and how agents co-configure these forms of integrated interactions in everyday experiences. Through a microlevel CEA of a video-recorded interaction, I examine what hybrid presence looks like and how it can manifest.

\section{The situation: "You are the mail reader - can you do without the computer?"}

While conducting fieldwork in a general practice, I observed three doctors in their clinic, at their affiliated nursing homes, and as they went on medical visits in the homes of patients. I had an ethnographic focus on how the doctors used, produced, and interpreted digital consultations embedded in these different medical and collaborative activities. The following case example comes from a day spent in the clinic, where I followed a middle-aged male doctor as he read and wrote digital consultations on a laptop in the clinic's breakroom. While the doctor (D1) is sitting and concentrating on reading and writing the digital consultations, another doctor (D2) enters the room and initiates small talk. However, their engagement with each other is asymmetric: D2 cheerfully walks around, puts on his sweater, and vividly gestures while looking at D1. Meanwhile, D1 continues to concentrate with a fixed gaze and a still body directed towards the computer screen while hitting the keyboard with rapid strokes. Though concentrating on the computer, D1 attends D2 verbally. Figure 1 shows an overview of the situation. 
Figure 1 Overview of the setting

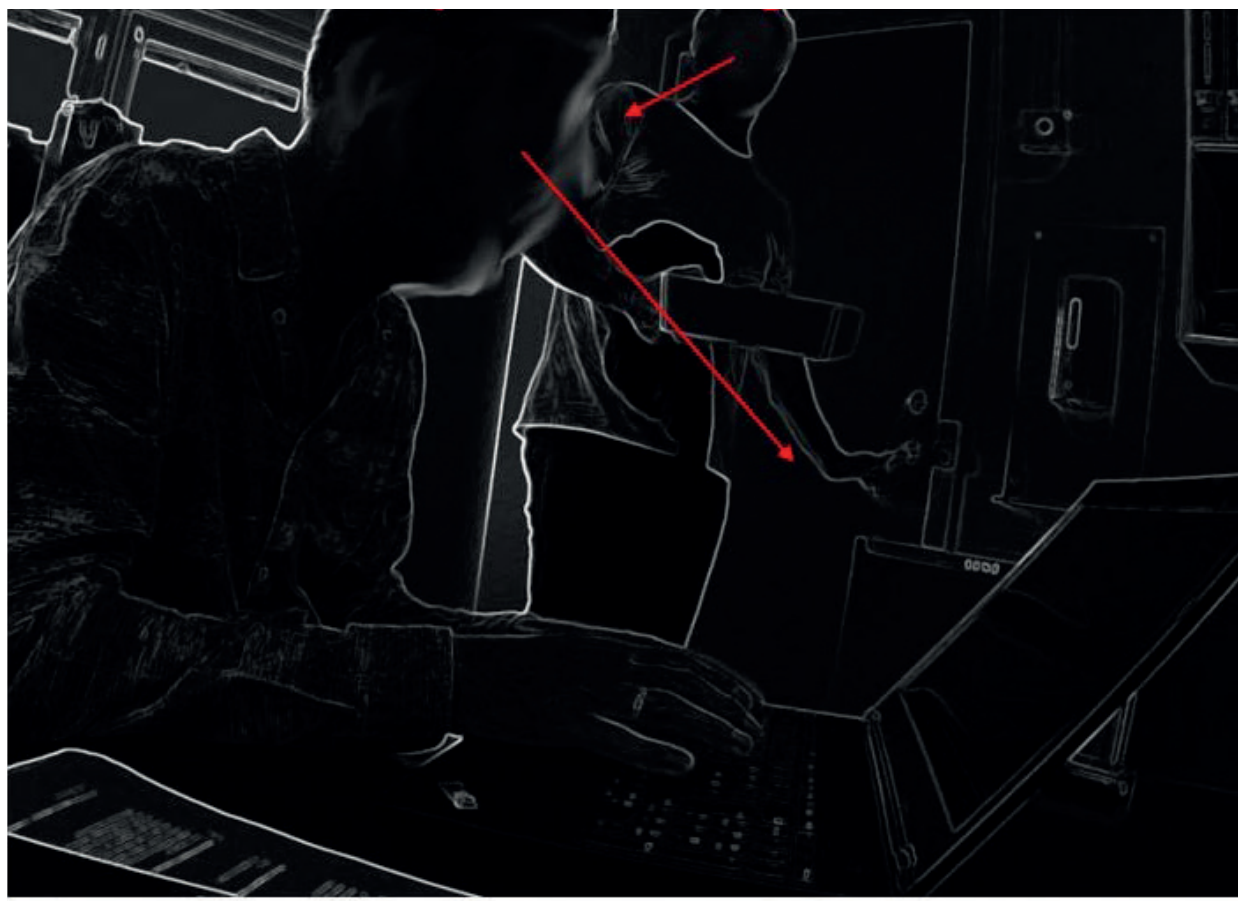

Comments: The first doctor (D1) sits in front of the laptop on the left, and the second doctor (D2) is the person standing a meter or two away at the door. On the one side, D1 has complete access to the digital consultations and to D2. On the other side, D2 must coordinate with D1 while D1 works with digital consultations. The figure shows the two agents directing their gazes in different directions, indicated by the red arrows.

At first glance, the doctors' discrepant engagement seems to indicate absent presence (Gergen, 2002), since D1 is preoccupied with the technology while D2 signals social engagement through his bodily micro-expressions (sighs, gaze, and posture directed to D1). However, due to what unfolds in the situation - namely that they are about to negotiate which of them can take the laptop computer - the case exemplifies how hybrid presence emerges because they jointly integrate D1's situated engagement with the digital consultations with the social interaction unfolding between them. Moreover, the reason for negotiating the use of the computer in the first place informs us of the importance of this collaborative instance; therefore, next is a deeper look at the context, followed by the microanalysis.

\section{How hybrid presence emerges: The problem-management event}

During the day, D1 has used the laptop, as he was in and out of the clinic several times and needed to be mobile while conducting medical home visits. Between visits, he returned to the clinic, where he sat in the breakroom and managed digital consultations. As he was running late on his last medical home visit, he needed to take the laptop with him after the clinic had closed. However, according to the clinic's procedural norms, D1 has no claim to take the laptop with him. Because the general practice has adapted to the Danish government's top-down requirement of implementing and providing digital consultations for patients, the doctors operationalised a mail-reader role in their practice, and this simply means that whoever is the designated mail reader has the claim to take the laptop computer. 
Recalling that, though the doctors can smoothly engage in the activity level of reading and writing digital consultations, the organisational level regarding the managerial work of administering the digital consultations requires a different kind of practico-medical work that is up to each individual clinic to manage. While all the doctors have access to the same digital consultations in a shared digital inbox - like exchanges from patients, nurses, and care assistants from nursing homes - it is the designated mail reader who is responsible for monitoring all the e-mails and attending to test results from laboratories and hospitals during the 14-day shift as the mail reader. If a test result is critical, then whoever has the responsibility as mail reader must immediately initiate corresponding medical actions, and if it is not critical, the mail reader must delegate the test results in the inbox, allowing the responsible doctor to communicate a proper response to their patient later. Thus, the mail reader is responsible for organisational taskwork and holds claim to the laptop, as this work often extends beyond the clinic's hours: "There are indeed extra hours in the weeks where you read mail; everyone knows that" (interview with a GP from the practice).

Though this practical constitution of the mail reader's responsibilities and corresponding claim to the laptop might seem simple, a problem emerges as D1 is not the designated mail reader but needs to take the computer with him. This situation makes an interesting cognitive event because the mail-reader role emerges as the radial point of the organisational structure of managing the digital consultations, which the two doctors must negotiate to solve the problem.

Accordingly, the problem cannot be reduced to a matter of simply negotiating hardware (and it is not comparable to the negotiation of any other material object). Instead, solving the problem includes a negotiation of the practitioners' organisation and use of the digital consultations: they are not just coordinating which of them ought to use the laptop; they are coordinating role responsibilities, and hence, the clinic's institutional structures that govern the overall management of digital work. In terms of CEA, this instance of problem management is thus identified as a pivotal cognitive event. The result of their interaction is that they solve the problem, arguably as they integrate the situated use of the technology with the emerging needs unfolding in the social interaction. Hence, the problem-management event demonstrates how they achieve hybrid presence.

The overall interaction is characterised by four salient transition phases, which are illustrated in Figure 2.

\section{Figure 2 The cognitive trajectory}

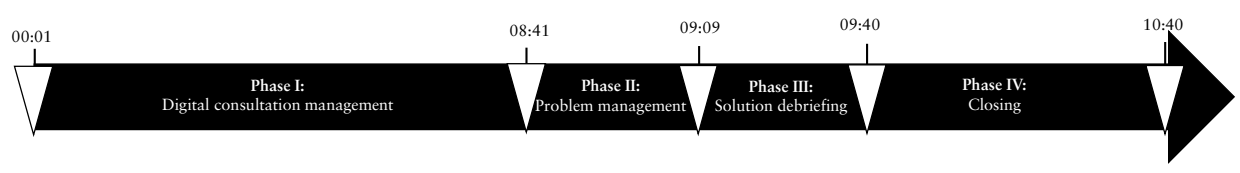

Comments: The white triangles illustrate the points in time where salient interactional transitions emerge. In Phase I, D1 manages digital consultations alone in the breakroom in silence. Phase II commences as D2 enters the room. Although D1 continues to manage digital consultations, he distributes his attention between these and D2 as he declaratively asks, "and you are the mail reader?" This prompts the two doctors to engage in the stage of problem management. In Phase III, the two doctors elaborate the solution they found in Phase II, and finally, in Phase IV, the interaction closes.

In Phase I, D1 is sitting alone in the breakroom, writing and reading digital consultations while simultaneously preparing for a medical home visit he is going to conduct after the clinic closes. In Phase II, prompted when D2 enters the breakroom, D1 shifts his attention from being completely absorbed in the digital environment to directing 
some of his attention to D2. Here, D1 pivotally and explicitly orients to D2 as "the mail reader" while still gazing at the computer and continuing to write and read digital consultations. In Phase III, the two doctors debrief solutions to the problem, as D2 suggests additional ways to help D1 with his upcoming medical home visit. In Phase IV, D1 disengages from the digital consultations and interacts with D2 about their work at a nursing home while D2 prepares to leave. They finish the interaction, having adjusted the organisational task-performance so D1 can take the laptop with him despite not being the designated mail reader. After this, D1 reengages with the computer and the digital consultations.

Because it was during Phase II (see Figure 2) that hybrid presence emerged, I zoom in on this interactional phase. In line with CEA, I have identified the event pivots (see Figure 3) that visualise changes in the problem-management event; D1 and D2 switch from problem presentation to solution probing and problem solving.

Figure 3 Event pivots in Phase II

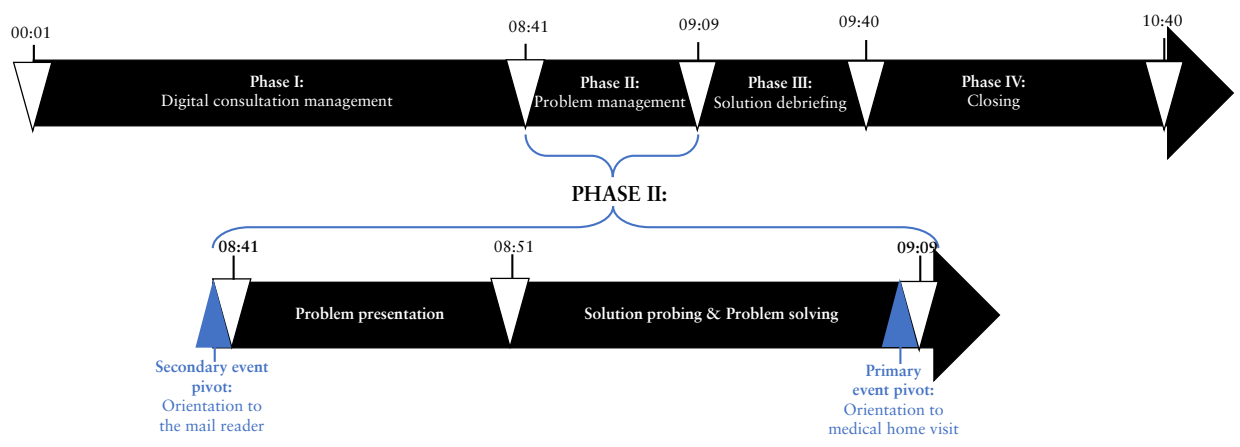

Comments: This figure illustrates transitions (white triangles) and event pivots (blue triangles), that is, the definitional transitions (in CEA terminology) that configure the cognitive trajectory of the problem management event in Phase II. As CEA works backwards and starts with the cognitive result, the primary event pivot marks the cognitive result of the interaction: when D1 pays attention to conducting a medical home visit with the laptop in his possession as a result of the problem management event. The opening event pivot is marked as secondary and indicates the point in time when D1 first orients to D2 as the mail reader. Between the event pivots, the phase undergoes a micro-interactional transition (white triangle), which indicates a change in attention from problem presentation to solution probing.

In the following microanalysis, I scrutinise the dialogical and cognitive processes that enable the doctors to solve the problem as they achieve hybrid presence.

\section{What hybrid presence looks like in interprofessional interaction}

The purpose of this and the next section is to gain an in-depth understanding of what hybrid presence looks like in the interprofessional interaction between the two doctors. I have characterised the first part of the interaction as a stage of problem presentation (see Figure 3): D1 indicates a need for the laptop while not being the designated mail reader, which in practice means that they have to reorganise the organisational taskperformance. Therefore, I first give an overview of this part of the interaction via the transcription (see Figure 4). As D2 enters the room (line 1), D1 immediately orients to him (line 2): 
Figure 4 Reorganising task-performance (lines 1-10)

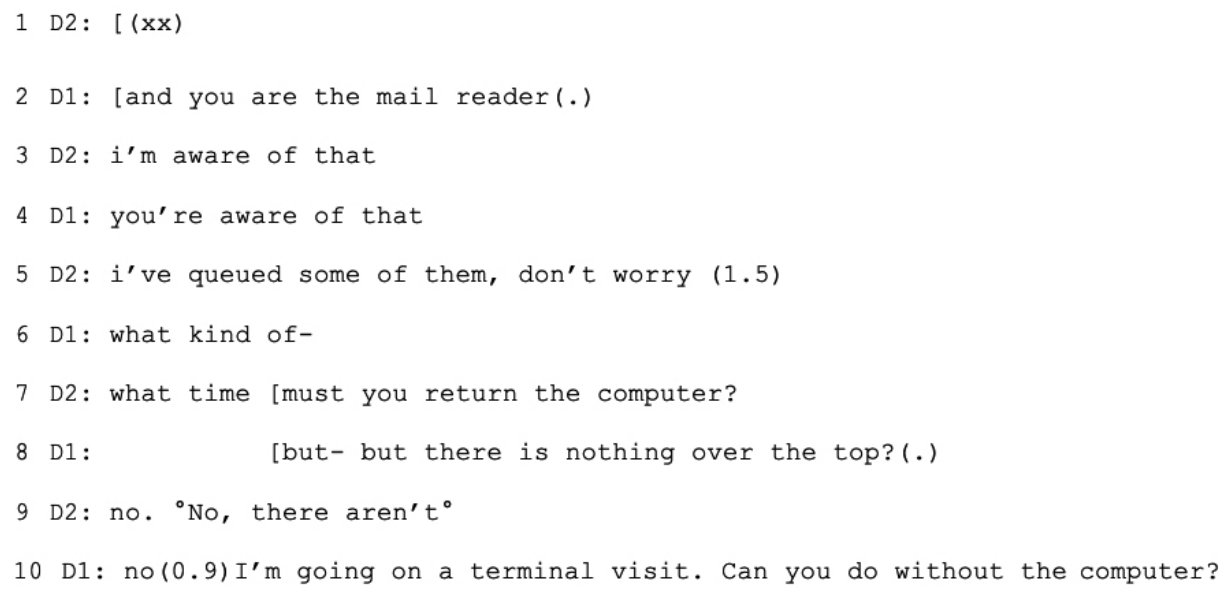

Comments: I have employed and adapted with minor changes the transcription conventions from Sacks and colleagues (1974, 1992): " $x x$ " in parentheses indicates the transcriber's uncertainty about the words articulated by the speaker; numerals in parentheses indicate the length of pauses in seconds; punctuation in parentheses indicates micropauses; an opening square bracket indicates the point in time when the speaker's talk is overlapped by another; a hyphen indicates the speaker's self-interruption; a degree sign $\left({ }^{\circ}\right)$ indicates when the speaker lowers his or her voice. All transcripts have been translated from Danish to English in collaboration with a professional English translation agency.

As D2 enters the breakroom, he initiates small talk but is interrupted by D1, who orients to D2 as "the mail reader" (line 1-2). In doing so, D1 integrates the present dialogue with their self-organised structure for managing digital consultations. This is evident, not only as D1 engages with D2 as a doctor or colleague, but as he simultaneously engages with the third party (Linell, 2017) nested in the reference "mail reader", it indicates the verbal invocation of institutional procedures and rules governing their medico-digital practices. Conducive for their collaboration, D2 replies with "i'm aware of that [...] i've queued some of them, don't worry (1.5)" (lines 3-5), which shows how D2 engages in the social interaction based on his role as the mail reader. This role enactment manifests in the way he articulates the technology-specific term "queue", which, on the one hand, is a dialogical invocation of features of the digital system, and on the other, practically works as a way to give D1 information about the status of the administrative tasks the mail reader must perform, namely that D2 had already delegated the noncritical test results.

Next, as D2 asks D1 “what time [must you return the computer?" (line 7), he marks his awareness of the problem at hand: they must share one computer. In doing so, he bases his dialogical engagement with the unfolding activity D1 performs, and his behaviour suggests involvement with D1 as opposed to introducing unrelated subjects, which could lead to absent presence. D2's dialogical involvement thus indicates an interpersonal integration between the two agents rather than a fragmentation.

However, before answering D2, D1 overlaps as he takes the mail reader's responsibilities into consideration, when he asks, "but- but there is nothing over the top? (.)" (line 8). By employing the metaphorical phrasing "over the top", D1 asks D2 if there are any critical test results necessitating immediate action, as "over the top" is 
a euphemistic way of saying "are there any red numbers in the system?" As D2 answers "no" (line 9), both doctors subsequently become aware that the mail reader's primary task has been conducted, and D1 is prompted to rephrase his question and ask permission for the computer: "I'm going on a terminal visit. Can you do without the computer? (1.3)" (line 10). Using the personal pronoun "you" instead of referring to "the mail reader" indicates that D1 is less engaged with the mail reader's tasks in this turn, which suggests how his dialogical engagement is directed with attention to D2. From a dialogical perspective, an interactional change indeed emerges as D1 shifts person deictic orientation (Bang et al., 2007), and in doing so, the deictic shift aids the transformation of how the two doctors organise meaning in their interpersonal engagement. In effect, D1 requests permission to take the computer from D2 as a person, colleague, or health practitioner, and not just as the mail reader. In practice, this interpersonal strategy allows for smoother face-preserving behaviour (Goffman, $1955,1959)$, as D2 has a different interagential status, than "the mail reader", who, in addition to representing a practical role, also represents the organisational power and structure of the entire technobureaucratic medical institution, which arguably may be more difficult to negotiate with. Accordingly, they immerse themselves in a similar organised dialogical engagement, which indicates that they are skilled at adapting to the needs emerging in the situation. This manifests in the way they smoothly adapt to each other's orientations, for example, as D2 engages in the situation with curiosity and interpersonal involvement. In doing so, he bridges with D1's requests throughout the sequence, despite their asymmetric access to the computer in use. D2 engages in the situation based on his ability to integrate and manage his dual role as an organisational member (the mail reader) and a healthcare practitioner (a doctor and D1's colleague). As they interpersonally invoke technological features ("queued", line 5) and their implications ("over the top", line 8), the doctors become interpersonally and cognitively involved in the social interaction symmetrically, despite D1's sole engagement with the computer and the digital consultations.

Their hybrid dialogical-cognitive abilities thus enable them to engage coherently in the situation and this phenomenon accordingly indicates hybrid presence: an integrated engagement with the technology and social interaction. While this part of their interaction relies on dialogical coordination, it is also possible to identify how hybrid presence is embedded in coordinated gestures, which appears in the next part of their interaction. In this final part of their interaction (see Figure 5) they probe for a solution and solve the problem (see Figure 3).

\section{Consequences for task-performance: Solving the problem via hybrid presence}

As D2 responds to D1's request (line 11), he allows D1 to take the computer, saying "All right, then (2.1)": 
Figure 5 Solving the problem (lines 11-19)

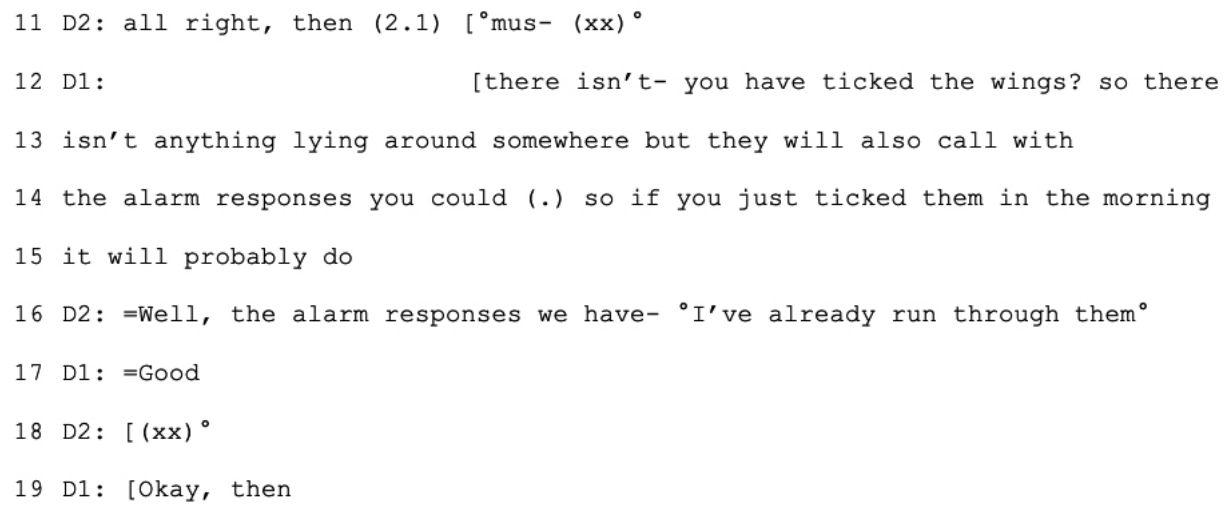

Comments: I have employed and adapted with minor changes the transcription conventions from Sacks and colleagues $(1974,1992)$ : "xx" in parentheses indicates the transcriber's uncertainty about the words articulated by the speaker; numerals in parentheses indicate the length of pauses in seconds; punctuation in parentheses indicates micropauses; an opening square bracket indicates the point in time when the speaker's talk is overlapped by another; a hyphen indicates the speaker's self-interruption; a degree sign $\left({ }^{\circ}\right)$ indicates when the speaker lowers his or her voice; and an equal sign indicates that there is no interval between the speakers' talk. All transcripts have been translated from Danish to English in collaboration with a professional English translation agency.

The practical value of D2's utterance is that it grants D1 permission to take the computer (line 11). However, the interpersonal delivery of it is dubious, as the utterance is technically not a direct preferred response (which would have been "yes"), as well as it is followed by a 2.1 second pause, which makes the response seem uncertain. However, instead of allowing such potential uncertainty to divide their negotiation and understanding of the situation, D1 invokes a technology-specific action related to the mail reader's tasks as he asks, "there isn't- you have ticked the wings? so there isn't anything lying around somewhere but they will also call with" (lines 12-13). "Ticking the wings" is a direct reference to the mail reader's task related to ticking boxes when assessing (potential) critical test results from the laboratory; when delegating the mail, the mail reader ticks the wings via the " $\sqrt{ }$ " symbol in the system. In addition to this dialogical invocation of the digital system, D1 physically embodies the symbol via a coordinated hand gesture (see Figure 6).

Figure 6 Ticking the wings

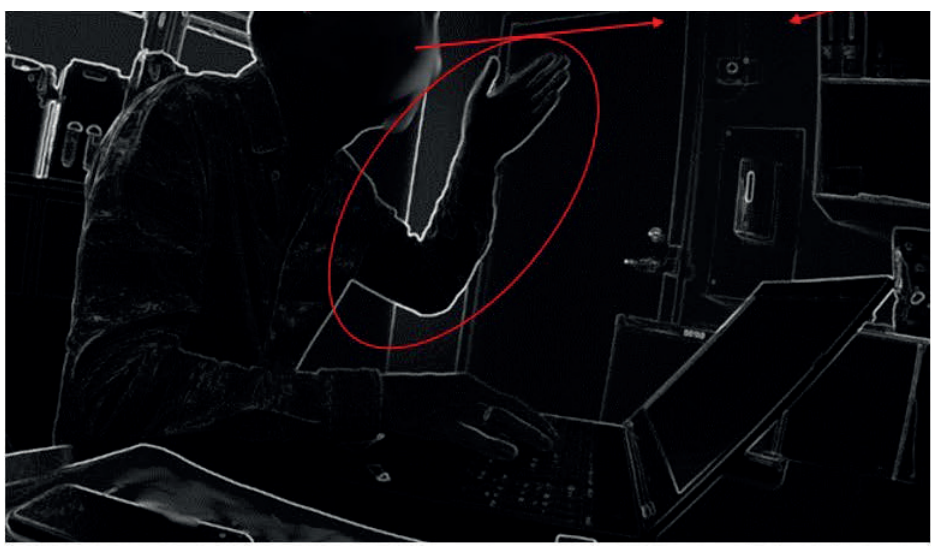

Comments: In this image, D1 (who sits at the laptop) gestures toward D2 (who stands out of the camera frame beyond the upper-right corner of the image) while synchronously verbalising the digital function "ticking the wings". D1's gesture works as a reenactment of a shape similar to the "V" symbol in the system. 
While D1 gazes at D2, he moves his hand in the shape of the symbol synchronously with his verbal utterance. In doing so, he embodies the digital system, and by gesturally reenacting (Alač, 2014) the semiotic feature in the social interaction with D2, he integrates the technical feature from the digital system into the social space of the interaction. Furthermore, this way of asking about the status of the mail reader's task-performance, is also a way of probing for a solution to the problem. Indeed, D1 introduces a suggestion for D2, as he elaboratively utters: "so if you just ticked them in the morning it will probably do" (lines 14-15). Accordingly, D1 ensures that the mail reader's responsibility is taken into account and that potential critical medical treatments are not neglected during this negotiation of organisational tasks.

The negotiation problem is solved as D2 replies "=Well, the alarm responses we have- 'I've already run through them ${ }^{\circ}$ " (line 16), thereby providing a functional answer that legitimises the solution to their problem: that D1 could take the computer since the mail reader's tasks were completed. The problem-management event is finalised when D1 states, "Okay, then" (line 19).

This CEA shows that because both doctors engage with each other in dialogical orientations that simultaneously consider D1's situated activity with the digital consultations and the sociotechnical organisation beyond the situated interaction, they sustain interpersonal coherence and promote certainty in the social interaction. This is evident in how D1 involves himself by addressing D2 as both the mail reader and colleague, and how D2 addresses the activity D1 performs with curiosity as he adaptively responds to D1's questions. Despite the apparent risk of absent presence (Gergen, 2002), such as the lack of eye contact and stiff body posture (Aagaard, 2016), the doctors collaboratively solved the problem. They reorganised the predetermined procedure for managing the digital consultations by invoking technological-specific orientations ("queue" and "ticking the wings") and matched them with the needs emerging in the social interaction ("you are the mail reader" and "Can you do without the computer?"). Thus, the result of their (hybrid) behavioural skills enables them to solve the problem as they successfully integrate the situated use of the technology with the dynamics of the social interaction.

\section{Discussion}

\section{Revisiting absent presence}

In this article, I have revisited Gergen's concept of absent presence, which describes a widely known struggle with technology, namely that using technology in social interactions divides presence and erodes a coherent sense of engagement and meaning (see Aagaard, 2016; Baym, 2015; Gergen, 2002). Conversely, some studies (e.g., Greatbatch et al., 1993, 1995; see Luff \& Heath, 2019; Nielsen, 2019) and empirical findings (presented in this article; Simonsen, in progress) suggests that healthcare practitioners often coordinate their technological and interpersonal engagement in ways that enable them to exploit resources from both technology and social interaction. Thus, my aim in this article was to recognise and explain this kind of conducive social behaviour involving technology and understand how healthcare practitioners' everyday experiences in the context of using, producing, and interpreting digital consultation technology may not be impaired by apparent asymmetric engagement with technology in the social interaction. 
The article is based on empirical observations, and in particular, I zoomed in on how microsocial dynamics emerged in a specific case of interprofessional interaction to explain how these ethnographic findings challenge Gergen's (2002) concept of absent presence.

Based on the ethnographic study and theoretical notions of hybridity, I proposed the concept of hybrid presence to explain how the agents establish coherent attention to what is going on despite of being otherwise engaged (D1 paid attention to the computer and D2; D2 paid attention to D1). Though the interaction is situated, the dynamics going into the coordination of their attention is multi-scalar (Gahrn-Andersen et al., 2019; Steffensen \& Pedersen, 2014) - that is, their interaction is constrained by more than one single time scale, as, for example, the preceding procedures for using the laptop relates to a slow, sociocultural scale, which work as a constraint on how they, on the another rapid, situated, and embodied scale, make sense of what is going on in the situation. The concept of hybrid presence unveils interactional complexities that challenge Gergen's concept of absent presence, which conversely suggests that interactions with technology necessarily induce fragmented presence and incoherent meaning due to the agents' divided attention (Gergen, 2002). However, this was not the case, as the agents not only adapted to each other dialogically, but moreover exhibited cognitive abilities that allowed them to negotiate and solve a problem. Accordingly, they collaboratively made sense of the situation, and co-created coherent meaning that allowed for a successful negotiation to happen. Therefore, it is relevant to discuss the extent of the concepts' validity and adequacy for interactions, as well as when, how, and why some interactions may result in hybrid presence while others result in absent presence.

\section{When, how, and why are absent presence and hybrid presence configured?}

Based on the empirical findings as well as the dialogical and distributed theoretical perspectives that emphasise an epistemological interest for interactional dynamics, I suggest the importance of recognising that absent presence does not per default emerge just because a technology is used by one participant during a social interaction. Interactions are not fragmented by default when technologies are used; rather, the degree of fragmentation (or integration) depends not only on the mere presence of technology, but the situated interaction with the technology and the copresent agents' abilities to involve themselves in the unfolding activity. Therefore, the questions of when, how, and why require scientific and methodological rigor to illuminate the particular interactional processes with which absent or hybrid presence is configured. Overall, interactions might result in absent presence when participants are indifferent to each other (Aagaard, 2016), which may be due to lacking microsocial dynamics (e.g., eye contact). Why some interactions lead to absent presence can perhaps be traced to conflicting or incompatible goals between participants. For example, one agent might need to search for medical information on a computer, while another might need to discuss an upcoming visit to a nursing home; subsequently, agents may divide their attention, signalling different engagement both dialogically and intercorporeally.

Conversely, interactions may result in hybrid presence when agents involve themselves in the situation by means of how they pay attention to what is going on. This leads me to suggest that coherent (hybrid) presence is not just determined by whether agents align in their bodily or dialogical engagement - whether they, for instance, 
have eye contact or not. Indeed, to identify hybrid presence, one must identify what the integrated behaviour looks like and how it develops. Thus, hybrid presence cannot be reduced to a simple observation or description of behaviour, such as identifying eye contact; rather, researching hybrid presence depends on investigating the interactional process that enables integration of cross engagement between social interaction and technology. For example, in the case study, the doctors managed the problem with the computer, engaging in the hybridity of the situation via verbal articulations and gestural reenactments that were linked with the situated use of the technology, which arguably led them to a collective understanding of what was going on and how to negotiate. Specifically, the practitioners' dialogical engagement and their degree of interpersonal involvement mattered. For instance, D2 spontaneously informed D1 that he had already queued some of the mails (Figure 4, line 5) without D1 asking, which exemplifies D2 involving himself in D1's activity with the computer in a way that was supportive of D1's engagement with the digital consultations. Moreover, D1 promoted certainty in the interaction, as he himself searched for a solution and rephrased his questions (Figure 5, lines 12-14), and the interaction resulted in hybrid presence because both agents ultimately included each other in the unfolding dynamics in the situation. Hence, interactions may result in hybrid presence when participants are aware of and become curious about the multiple goals, needs, and tensions that constrain and unfold in a situation. Why some interactions result in hybrid presence may be due to agents' habituated patterns of using technologies in particular settings, as well as the care, awareness, and interest between the participants.

Subsequently, discussions of empirical adequacy ought to be motivated from an empirical investigation of participants' situated behaviour and salient contextual features. An empirically oriented investigation promotes inferences of either absent or hybrid presence; by means of empirically derived observations or theoretically informed observer-dependent categorisations, one can investigate interactional processes and thus identify how the interactions may result in integration (hybrid presence) or fragmentation (absent presence). It can therefore also be considered a shortcoming that neither Gergen (2002) nor Aagaard (2016) included interactional analyses in their work, though they both promoted critical inferences related to face-to-face communities. However, this article is not without its own limitations.

\section{Limitations}

An apparent limitation of this article relates to its ethnographic origin. While I make inferences from a behavioural-observant point of view, concluding that the agents aligned and integrated their interaction and achieved hybrid presence, the microanalysis was mostly built on observer-dependent criteria informed by theory and ethnographic observations that were focused on the usage of digital consultations and did not originate from an explicit research focus on absent presence. This means that though the practitioners' behaviour suggests integrated coordination, D2 may still have experienced D1 as being absent present despite my analytical inferences. If one participant feels like the other is absent present, the interaction is arguably impaired. However, the ethnographic study (Simonsen, in progress) was organised around detecting the emergence of hybridity in interprofessionals' use, production, and interpretation of digital consultations, 
which has guided a conceptualisation of hybrid presence that indeed challenges absent presence and, at least philosophically, aids how one can recognise such complexities in future investigations.

\section{Conclusion}

The concept of hybrid presence allows us to see and understand how technologies may be integrated by practitioners in their everyday interprofessional interactions through language, roles, organisational structures, habits, and social collaborations. This observation challenges Gergen's (2002) conceptualisation of absent presence in relation to the usage of digital consultation technology in interprofessional interactions, thereby encouraging us to understand and explain the multifaceted usage of technologies in social interactions.

On a final note, it is relevant to consider how Gergen's (2002: 229) conceptualisation of absent presence pertains to "virtually all communications technologies that enabled people to communicate at a distance". Throughout, Gergen describes both functional and abstract ways in which technology challenges coherent meaning-making in face-to-face communities, from the way individuals engage with absent voices when absorbed with print technology to more dynamic and interactive instances, such as when one engages with a presence that is "virtually eradicated by a dominating absence" (Gergen, 2002: 231) when one writes an e-mail. Accordingly, Gergen argues, on the one hand, as people engage more with technology and the multiple meanings technology carries, coherence and sustained presence in face-to-face communities is diminished (Gergen, 2002). On the other hand, when people engage with these meanings in face-to-face interactions, the social copresence of the individuals and collective coherence are undermined as people subsequently divide their attention between technology and the copresent other. However, while Gergen's arguments raises important critical notions pertaining to the development of communication technologies and the uses of technology in social interactions (see Aagaard, 2016; Gergen, 2002), less is known regarding the particular social contextualisations and specific micro-interactional processes in which technologies are embedded. This article has demonstrated that much can be gained by considering such empirical crystallisation, as the empirical findings suggest that it is indeed possible for practitioners to use technology as an integrated part of their social interactions. Accordingly, the concept of hybrid presence offers a way to understand and explain the micro-interactional processes that enable practitioners to use a technological artefact as part of their interaction and engage with the technology-induced meanings (e.g., "ticking the wings") in ways that allows them to sustain coherence and collaboration in making sense of the social interaction.

Overall, the ethnographic findings and CEA have shown that the practitioners' social interactions do not appear impeded by the mere presence of technology, and the concept of absent presence did not manifest as a technological struggle in the everyday experiences of the practitioners. However, although the doctors solved the computernegotiation problem, other technology-induced struggles could exist, which can be tied to the dynamically conditioning presence of third parties (Linell, 2009). This is evident in the administrative and managerial workload induced as part of the implementation of new digital media, which was the main reason that the practitioners even had to 
institutionalise the mail-reader role in their practice in the first place. The modern-day technological struggle is thus importantly a matter of illuminating the normative preconditions that practitioners must deal with when technology is implemented in practice, as these normativities indeed influence the organisation of the social interaction. Therefore, it is crucial that researchers in media, sociology, linguistics, anthropology, healthcare communication, and cognitive studies consider how technologies may afford multiple meanings and how they potentially are integrated in interactional processes, as these shape everyday experiences and how agents make sense of social interactions.

The microlevel analysis presented in this article ought not be thought of as mere academic pedantry; rather, it is an essential and qualifying precondition for future improvements in, for example, the education of healthcare professionals and the sociomaterial organisation of healthcare practices: The value of the (CEA) investigation is indeed that we get an in-depth and nonreductionistic understanding of human behaviour (Trasmundi $\&$ Steffensen, 2016: 178) with technology and the rules and social practices that organise how we use it, whether we recognise it or not, at the manifest level of consciousness. By analysing a seemingly mundane everyday interaction in the working lives of two doctors, latent and ambiguous conditions for performing and organising medical work with digital consultation technology are made visible that would otherwise comprise hidden yet dynamically conditioning dimensions of practice and professional behaviour, unavailable for reflection and, hence, improvement. The practical value thus lies in the fact that knowledge and scientific symbolisation of the demands and conditions inherent to the current (digitalised) landscape of medical and healthcare professions are an essential requirement if we wish to be able to 1) recognise how technology may work as an organising part of interprofessional interactions and the organisational structures and roles embedded in everyday experiences and collaborative situations in medical work; and 2) educate future healthcare professionals with awareness of the challenges and possibilities of absent and hybrid presence that accompany technological embeddedness in social interactions; which can ultimately 3) aid a gradual improvement of the organisation of healthcare practices by supporting practico-structural conditions that successfully enable rather than inhibit the processual embodying of hybrid presence among practitioners.

\section{Acknowledgements}

The present study was supported by a grant from the VELUX FOUNDATIONS [grant number 18158].

\section{Notes}

1. While Gergen (2002) primarily emphasises examples related to mobile phone use, he also notes that absent presence is a phenomenon that characterises all kinds of communication technologies'-including print technology - dividing potentials.

2. Digital consultations (or digital correspondences, e-mail consultations, e-consultations, or econs) include text exchanges, including the option of attaching photos and sending test results, prescription renewals, and consultation booking.

3. Participants were recruited as part of my research project's affiliation with a larger research project about digital consultations (2018-2021) (Grønning, 2016). The larger research project (Grønning, 2016) consists of five subprojects that all investigate different perspectives on the usage and experience of digital consultations.

4. I do not claim that absent presence never emerges in the context of interacting with digital consultations, nor that the practitioners' interactions are always coherent and functionally integrated when they are using 
technology. Rather, in this article, I am interested in what integrated interprofessional interactions look like, how they counter Gergen's conceptualisation, what such cases looks like, and how we can understand them as examples of integration by the practitioners' use of other agents, technology, and organisational context.

\section{References}

Aagaard, J. (2016). Mobile devices, interaction, and distraction: A qualitative exploration of absent presence. Ai \& Society, 31(2), 223-231. https://doi.org/10.1007/s00146-015-0638-z

Alač, M. (2014). Digital scientific visuals as fields for interaction. In C. Coopmans (Ed.), Representation in scientific practice revisited (pp. 61-87). MIT Press.

Alač, M., \& Hutchins, E. (2004). I see what you are saying: Action as cognition in fMRI brain mapping practice. Journal of Cognition and Culture, 4(3-4), 629-661. https://doi.org/10.1163/1568537042484977

Assing Hvidt, E., Søndergaard, J., Klausen, M., \& Grønning, A. (2020). Not just an information-delivery tool: An ethnographic study exploring Danish GPs' perspectives on and experiences with the relational potential of email consultation. Scandinavian Journal of Primary Health Care, 38(4), 411-420. https:// doi.org/10.1080/02813432.2020.1843939

Bang, J. C., Døør, J., Steffensen, S. V., \& Nash, J. (2007). Language, ecology, and society: A dialectical approach. Continuum.

Bakhtin, M. M. (1981). The dialogic imagination: Four essays (C. Emerson, \& M. Holquist, Trans.). University of Texas Press.

Baym, N. K. (2015). Personal connections in the digital age. John Wiley \& Sons.

Becvar, L. A., Hollan, J., \& Hutchins, E. (2005). Hands as molecules: Representational gestures used for developing theory in a scientific laboratory. Semiotica, 156(1/4), 89-112. https://doi.org/10.1515/ semi.2005.2005.156.89

Chemero, A. (2000). What events are. Ecological Psychology, 12(1), 37-42. https://doi.org/10.1207/ S15326969ECO1201_3

Cowley, S. J., Major, J. C., Steffensen, S. V., \& Dinis, A. J. (2010). Signifying bodies: Biosemiosis, interaction and health. Catholic University of Portugal.

Fage-Butler, A. M., \& Jensen, M. N. (2015). The relevance of existing health communication models in the email age: An integrative literature review. Communication \& Medicine, 12(2-3), 117-128. https://doi. org/10.1558/cam.18399

Gahrn-Andersen, R., Johannessen, C. M., Harvey, M. I., Simonsen, L. M., Trasmundi, S. B., Marchetti, E., Warsøe, L. B., Fester-Seeger, M.-T., Lebahn, M., \& Steffensen, S. V. (2019). Interactivity: Why, what and how? Rask, 50, 113-136.

Gergen, K. (2002). The challenge of absent presence. In M. Aakhus, \& J. Katz (Eds.), Perpetual contact: Mobile communication, private talk, public performance (pp. 227-241). Cambridge University Press.

Gibson, J. J. (1986). The ecological approach to visual perception: Classic edition. Psychology Press.

Goffman, E. (1955). On face-work: An analysis of ritual elements in social interaction. Psychiatry: Journal for the Study of Interpersonal Processes, 18(3), 213-231. https://doi.org/10.1080/00332747.1955.11023008

Goffman, E. (1959). The presentation of self in everyday life. Penguin.

Goffman, E. (1974). Frame analysis: An essay on the organization of experience. Harvard University Press.

Goffman, E. (1983). The interaction order: American Sociological Association, 1982 presidential address. American Sociological Review, 48(1), 1-17. https://doi.org/10.2307/2095141

Goodwin, C. (2013). The co-operative, transformative organization of human action and knowledge. Journal of Pragmatics, 46(1), 8-23. https://doi.org/10.1016/j.pragma.2012.09.003

Greatbatch, D., Heath, C., Campion, P., \& Luff, P. (1995). How do desk-top computers affect the doctor-patient interaction. Family Practice, 12(1), 32-36. https://doi.org/10.1093/fampra/12.1.32

Greatbatch, D., Luff, P., Heath, C., \& Campion, P. (1993). Interpersonal communication and human- computer interaction: An examination of the use of computers in medical consultations. Interacting with Computers, 5(2), 193-216. https://doi.org/10.1016/0953-5438(93)90018-O

Grønning, A. (2016). Digital consultations and ageing: Perceptions, mediation and ethics [Application for THE VELUX FOUNDATIONS, Odense, University of Southern Denmark].

Grønning, A., Assing Hvidt, E., Brøgger, M. N., \& Fage-Butler, A. M. (2020). How do patients and general practitioners in Denmark perceive the communicative advantages and disadvantages of access via email consultations? A media-theoretical qualitative study. BMJ Open, 10(10), e039442. http://dx.doi. org/10.1136/bmjopen-2020-039442.

Hollan, J., Hutchins, E., \& Kirsh, D. (2000). Distributed cognition: Toward a new foundation for humancomputer interaction research. ACM Transactions on Computer-Human Interaction, 7(2), 174-196. https://doi.org/10.1145/353485.353487

Hutchins, E. (1995). Cognition in the wild. MIT Press. 
Hutchins, E. (2014). The cultural ecosystem of human cognition. Philosophical Psychology, 27(1), 34-49. https://doi.org/10.1080/09515089.2013.830548

Latour, B. (2000). When things strike back: A possible contribution of 'science studies' to the social sciences. British Journal of Sociology, 51(1), 107-123. https://doi.org/10.1111/j.1468-4446.2000.00107.x

Laursen, D., Simonsen, L. M., \& Grønning, A. (under review). Methodological challenges in researching email consultations as a form of communication in patient-provider interactions. Qualitative Research.

Linell, P. (2009). Rethinking language, mind, and world dialogically. Information Age Publishing.

Linell, P. (2010). Communicative activity types as organisations in discourses and discourses in organisations. In S.-K. Tanskanen, M.-L. Helasvuo, M. Johansson, \& M. Raitaniemi (Eds.), Discourses in interaction (pp. 33-59). John Benjamins Publishing Company.

Linell, P. (2017). Dialogue, dialogicality and interactivity: A conceptually bewildering field? Language and Dialogue, 7(3), 301-335. https://doi.org/10.1075/ld.7.3.01lin

Linell, P., \& Thunqvist, D. P. (2003). Moving in and out of framings: Activity contexts in talks with young unemployed people within a training project. Journal of Pragmatics, 35(3), 409-434. https://doi. org/10.1016/S0378-2166(02)00143-1

Luff, P. K., \& Heath, C. (2019). Visible objects of concern: Issues and challenges for workplace ethnographies in complex environments. Organization, 26(4), 578-597. https://doi.org/10.1177/1350508419828578

Mol, A. (2002). The body multiple: Ontology in medical practice. Duke University Press.

Mondada, L. (2018). Multiple temporalities of language and body in interaction: Challenges for transcribing multimodality. Research on Language and Social Interaction, 51(1), 85-106. https://doi.org/10.1080/ 08351813.2018 .1413878

Nielsen, S. B. (2019). Making a glance an action: Doctors' quick looks at their desk-top computer screens. Journal of Pragmatics, 142: 62-74. https://doi.org/10.1016/j.pragma.2018.12.021

Norris, S. (2004). Analyzing multimodal interaction: A methodological framework. Routledge. https://doi. org/10.4324/9780203379493

Oudshoorn, N. (2009). Physical and digital proximity: Emerging ways of health care in face-to-face and telemonitoring of heart-failure patients. Sociology of Health \& Illness, 31(3), 390-405. https://doi. org/10.1111/j.1467-9566.2008.01141.x

Pedersen, S. B. (2015). The cognitive ecology of human errors in emergency medicine: An interactivity-based approach [Doctoral dissertation, University of Southern Denmark].

Pedersen, S. B., \& Steffensen, S. V. (2014). Temporal dynamics in medical visual systems. Cybernetics and Human Knowing, 21(1-2), 143-157.

Sacks, H., Jefferson, G., \& Schegloff, E. A. (1992). Lectures on conversation. Blackwell.

Sacks, H., Schegloff, E. A., \& Jefferson, G. (1974). A simplest systematics for the organization of turn-taking for conversation. Language, 50(4), 696-735. https://doi.org/10.2307/412243

Sarangi, S. (2000). Activity types, discourse types and interactional hybridity: The case of genetic counselling. In S. Sarangi, \& M. Coulthard (Eds.), Discourse and social life (pp. 1-27). Longman.

Scolari, C. A. (2012). Media ecology: Exploring the metaphor to expand the theory. Communication Theory, 22(2), 204-225. https://doi.org/10.1111/j.1468-2885.2012.01404.x

Simonsen, L. M. (in progress). Digitalization of the wild: A cognitive ethnography of interprofessional interactions with digital consultations [Doctoral dissertation, University of Southern Denmark].

Simonsen, L. M., \& Steffensen, S. V. (2021/in press). Hybrid cognition in medical discharges. International Journal of Organization Theory \& Behavior.

Simonsen, L. M., \& Steffensen, S. V. (2019). Hybrid cognition in medical simulation: Investigating microlevel organisational cognition. Proceedings of the European Academy of Management Conference, Lisbon, Portugal, 1-35.

Steffensen, S. V. (2012). Care and conversing in dialogical systems. Language Sciences, 34(5), 513-531. https://doi.org/10.1016/j.langsci.2012.03.008

Steffensen, S. V. (2013). Human interactivity: Problem-solving, solution-probing and verbal patterns in the wild. In S. J. Cowley, \& F. Vallée-Tourangeau (Eds.), Cognition beyond the brain: Computation, interactivity and human artifice (pp. 195-221). Springer.

Steffensen, S. V. (2015). Distributed language and dialogism: Notes on non-locality, sense-making and interactivity. Language Sciences, 50, 105-119. https://doi.org/10.1016/j.langsci.2015.01.004

Steffensen, S. V., Vallée-Tourangeau, F., \& Vallée-Tourangeau, G. (2016). Cognitive events in a problemsolving task: A qualitative method for investigating interactivity in the 17 animals problem. Journal of Cognitive Psychology, 28(1), 79-105. https://doi.org/10.1080/20445911.2015.1095193

Sutton, J. (2012). Exograms and interdisciplinarity: History, the extended mind, and the civilizing process. In R. Menary (Ed.), The extended mind (pp. 189-225). MIT Press.

Trasmundi, S. B. (2020). Errors and interaction: A cognitive ethnography of emergency medicine (Vol. 309). John Benjamins Publishing Company. 


\section{Line Maria Simonsen}

Trasmundi, S. B., \& Steffensen, S. V. (2016). Meaning emergence in the ecology of dialogical systems. Psychology of Language and Communication, 20(2), 154-181. https://doi.org/10.1515/plc-2016-0009

Tribble, E., \& Sutton, J. (2011). Cognitive ecology as a framework for Shakespearean studies. Shakespeare Studies, 39, 94-103.

(C) 2021 Nordicom and respective authors. This is an Open Access work licensed under the terms of the Creative Commons Attribution-NonCommercial-NoDerivatives 4.0 International Public licence (CC BY-NC-ND 4.0). To view a copy of the licence, visit https://creativecommons.org/ licenses/by-nc-nd/4.0/ 